\title{
Cultural Characteristics in Idiom Translation
}

\author{
Yu Fang* \\ School of International Education, Chifeng University, Chifeng, China
}

\begin{abstract}
Language is the carrier of culture and the idioms are the essence of it. In normal cultural communication, we often encounter the idioms with distinctive national cultural features and their application and translation problems. In the translation of idioms, we must take into account the customs and characteristics of ethnic cultures in different countries, needing to be sensitive to cultural differences between idioms and understand their cultural connotation and practical meaning to make translation vivid and faithful so as to make effective communication in different cultural environments.
\end{abstract}

Keywords-Intercultural communication; Idiom translation; Cultural characteristics; Expression relationship

\section{INTRODUCTION}

The term "idiom" is originated from Greek. It can be regarded as the essence of language - the carrier of culture. Therefore, the typicality of idioms is more pronounced than other language elements in expressing the cultural characteristics of language. Idioms are heavily culturally loaded phrases or sentences, which are usually highly specialized in meaning and images tied to distinctive cultural features. According to A Modern Chinese-English Dictionary (2001), Chinese idiomatic expressions are phrases or short sentences that are brief and fixed in form, concise and expressive in meaning. So idioms in broad sense may include: set phrases, proverbs, sayings, slang expressions, colloquialisms, two-part allegorical sayings.

Broadly speaking, the coverage of idioms in English and Chinese is roughly the same. English idioms include fixed word collocations (phrase), proverbs, motto and some slang while Cihai (1989 edition) said that Chinese idioms include phrase, proverbs, motto, locution, two-part allegorical saying, etc. English and Chinese idioms have their common characteristics in nature. They are all fixed and unchangeable phrases or sentences that have been formed in the long-term use of language. Chinese idioms also include two-part allegorical saying. The two-part allegorical saying can't find its corresponding expression method in English and the cultural connotation expressed by it is unique to Han nationality culture. Therefore, people must adopt other remedial measures to translate exactly [1].

The cultural characteristics of idioms can be inseparable from its rich and colorful sources. The different geographic conditions, unique literary works, different living habits and different thinking concepts of China and Britain have become the source to produce a large number of vivid and lively idioms. Sometimes, some idioms show some similar cultural characteristics because of the similar life experience and ideological understanding of the two nations. Cultural exchanges between the two countries also add some fresh idiomatic expressions to the two languages. However, this is only a small part of idioms, and a large number of idioms show different language habits and cultural characteristics.

In intercultural communication, we often encounter the idioms with distinctive national cultural features and their application and translation problems. In fact, idiom translation is a question of how to deal with various cultural characteristics expressed by idioms of source language in the target language. In cross-cultural communication, the relationship type of idioms in expressing cultural characteristics can be divided into corresponding relationship, semi-corresponding relationship and non-corresponding relationship.

\section{CORRESPONDING RELATIONSHIP}

Corresponding relationship means that sometimes English idioms and Chinese idioms are corresponding in whatever meaning or image. This is because there are some similarities between the two cultures. Idioms that express these similar content coincide with each other in meaning and image. In that case, the inter-translation between the two languages can be made. That is, the idioms of one language can be translated by the corresponding idioms of the other [2].

For example, the "burn one's boat" in English and the "Po $\mathrm{Fu}$ Chen Zhou" in Chinese both mean that cutting off all means of retreat to show one's determination to press ahead. When Julius Caesar led his army to cross the Rubicon, he gave the order to burn the boat to show the determination that if he doesn't win then he will die. When the King of Western Chu XiangYu crossed the river, he also gave the order to burn the boat to show his determination to win. The image and meaning of them are so similar, so we can use the method of inter-translation to deal with the written language communication. For example, "If you work conscientiously you'll only die pauper. All you can do is to burn your boats and fight them in the hope that one day you'll come out on top." "Ni an bu jiu ban de gan, zuo dao lao ye shi qiong si. Zhi you da dan de po fu chen zhou de gen ta men pin, cai you fan shen de na yi tian."

Similarly, for another pair of idioms in English and Chinese "Walls have ears" and "Ge Qiang You Er", they are also similar in the image and meaning. It is said that during the reign of Queen Catherine, some special walls were built in Louvre, and the voices in one room could be heard clearly in another room. The suspicious queen knew many state secrets and the conspiracies of some people by this way [3]. The 
idiom "Walls have ears" in English comes from here. The idiom "Ge Qiang You Er" in Chinese comes from "Guan ZiJun Chen Xia": "Gu zhe you er yan: qiang you er, fu kou zai ce," "Qiang you er zhe, wei mou wai xie zhi wei ye." Therefore, the two idioms both mean that the secret talk is overheard. With the same meaning and image, translation can be regarded as the inter-translation between equivalent idioms. For example, "Shall I come to y our room?" "No. Walls have ears. We'd better meet in the woods." "Wo dao ni fang jian lai hao ma?" "Bie lai, zan men zui hao dao shu lin li mian, xiao xin ge qiang you er."

In addition, some idioms in one language are borrowed from another language. Borrowed idioms enrich the expression of this language and have been used as the constant idioms in this language over time. These borrowed idioms still retain the image and meaning of the original idioms, so they all display the same cultural characteristics. For example, the idioms "Yi Yan Huan Yan, Yi Ya Huan Ya", "Wu Zhuang Dao Ya Chi" and "Pi Zhe Yang Pi De Lang" in Chinese are borrowed from English idioms "an eye for an eye, a tooth for a tooth", "armed to the teeth" and "a wolf in sheep's clothing." The idioms "to lose one's face" and "to save one' s face"in English are borrowed from Chinese idioms "Diu Mian Zi" and "Bao Mian Zi."

Thus it can be seen that there are some equivalent idioms in English and Chinese. They use the same figures of speech, display the same cultural image and express the same meaning in literalness and connotation. Some of them have the same grammatical function, and some simply come from the same source. These idioms show the corresponding and consistent place in many aspects, therefore, they become the corresponding relationship when they display the cultural characteristics [4]. But this coincidence and borrowing are rare.

\section{SEMI-CORRESPONDING RELATIONSHIP}

Though human beings enjoy great similarities in their thin king and languages, their cultures are alarmingly different. Th ey have different history, belief value, and modes of thinking and living habits, as leads to the partial equivalence of the idio ms. Most idioms belong to semi corresponding relationship or non corresponding relationship. Semi-corresponding relationship is also called partial corresponding relationship. In the semi-corresponding idioms, some have the same meaning but have the different figures of speech, so the image of them is also different. Some idioms with similar meaning are used in different occasions or contexts. Some have the similar literal meaning but have different connotations and emotional colors. Therefore, they just express partial corresponding cultural characteristics. When translating these idioms, we can use the corresponding idioms, but in more cases, we should flexibly deal with them according to the meaning of the contexts [5].

If you want to express the meaning that two people have the same shortcoming or mistake, or someone accuses others but he made the same mistake, you can use "the pot calls the kettle black" in English and use "Wu Shi Bu Xiao Bai Bu" in Chinese. For example, "When he blames her for wasting money, he conveniently forgets that he regularly loses half of his own wage by betting on the wrong horses -it is the pot calling the kettle black, I'd say." "Ta yi mian ze bei ta luan hua qian, yi mian que ji hu wang le ta zi ji du sai ma chang chang du shu qian, ba zi ji de yi ban xin shui dou shu diao le-wo shuo zhe shi zai shi wu shi bu xiao bai bu."

However, although they are similar in meaning, they can not make the inter-translation in any case. The meaning of this Chinese idiom is that "two people have mistakes, but one is lighter than the other." However, there is no latter meaning in English.

Therefore, the translation should adjust the original idioms according to the context. For example, "Jason: "Marry me, now, Sonia, we have witnesses, we could become one here and now."

\section{Sonia: "Don't be a dirty old man, you dirty old man."}

Jason: "Huh, the pot calls the kettle black."

Jie Xun: "Xian zai jiu jia gei wo ba. Suo Niya, wo men lai xie ai qing de jian zheng, wo men ke yi xian zai zai zhe er he wei yi ti."

Suo Niya: "Lai dian zheng jing de hao bu hao, ni zhe lao se gui."

\section{Jie Xun: "You he, wu ya dao xian qi qi zhu hei la."}

From the above examples, we can see that the basis of inter-translation is the meaning of idioms. When the connotation and meaning are the same, we can choose the most appropriate and the most natural idioms of targeted language to translate the original idioms. However, most idioms are only similar at one point in meaning or image and they have other distinctive features [6]. Translation is unreliable according to the superficial phenomenon. The value of idioms first lies in their meanings. The basis of intertranslation should be based on the similarity of meaning.

\section{NON-CORRESPONDING RELATIONSHIP}

Biguenet, J. and Scheulte, R said that a cultural situation whether in the realm of social, ethical, educational, legal, or, political realitiesnever finds its exact equivalent in another co untry. Different geographic locations of the English and Chine se people lead to differences in thinking, images and metapho rs, so it is often found that cultural messages and their images conveyed by target idioms are totally different from those in $t$ he source idioms. Idioms with non-corresponding relationship refer to the idioms with nothing in common whatever in meaning, usage or cultural characteristics. This kind of idiom can't be translated literally. They have their own meanings and usage, which express different cultural colors. Some idioms are similar in literal image, but they are not the corresponding idioms. The meaning and cultural characteristics of them are totally different, such as "child's play" and "Er Xi." The literal image of them is almost the same, however, their meanings are totally different. In English, "child's play" means something very easy to do, something not very important. The "Er Xi" in Chinese is used to mean 
that someone is not responsible or careful for an important job or thing. The two are totally different, so they can't make the inter-translation.

We often encounter idioms with similar literal meanings, which are very easy to form a translation trap. For example, for the English idioms "eat one's words", we are easy to think of the Chinese idiom "Shi Yan" or "Shi Yan Er Fei" because of the similar literal image of them. However, in English, "eat one's words" means that someone takes back what he said and admits what he said are wrong. It has a certain emotional color and is shameful. If someone has to "eat his words", he should "retract them in humiliating manner" (The Concise Oxford Dictionary 1980 edition). In Chinese, "Shi Yan" means that someone breaks his promise. Therefore, there is nothing in common between the two idioms, and the direct translation can be impossible [7]. For example, "after all his boasting that he would drive the other company out of business, Mr. Angus had to eat his words for the other company flourished more than his did." "An ge si xian sheng ceng da yan bu can de chui xu yao jiang ling yi jia gong si ji kua, dan na jia gong si bi ta men de gong si geng xing wang, ta zhi hao can kui de cheng ren qian yan you shi." From it, we can see that the meanings of "eat one's words" and "Shi Yan" and "Shi Yan Er Fei" are totally different.

\section{CONCLUSION}

In conclusion, due to the different sources, idioms of different countries inevitably express different cultural features, customs and characteristics. The idioms in this culture may be a blank in that culture. The same meaning, in this culture, such an expression, which may be entirely another expression in that culture. Therefore, in the translation of idioms, we must take into account the different customs and characteristics of different national cultures and need to be sensitive to cultural differences between idioms. It can be said that cultural diversity limits the translatability of idioms. Idiom translation can only be carried out within a certain range. This range varies according to the varying degrees of the attachment of idiom to the culture. For those idioms with strong cultural color, it is difficult to translate them vividly. Language is the carrier of culture. Communicative language has the problem of understanding and expressing cultural connotations and these problems mainly exist in intercultural communication. Idiom translation is the prominent manifestation of the contradiction of the understanding and expressing in intercultural communication. In order to solve this contradiction, translators must try to improve their crosscultural sensitivity. This requires the translator not only to have bilingual ability, but also to have dual-cultural and even multi-cultural knowledge, so as to meet the needs of effective communication in different cultural environments.

Idioms, as part of language, are usually characterized by concise and rich language, and vivid and humorous expression. They generally refer to the fixed phrases and short sentences extracted from the long use the language, mainly including religious belief, geography and living convention. However, it is difficult to understand and use idioms correctly. The proper use of idioms generally represents the ability of a person to control the language. In the speech or writing, the use of idioms will enrich the content of the article, and make the article full of color, which can better attract audiences. However, the inappropriate use or overuse will eclipse the article. So people often say that the degree of the mastery of idioms represents a person's language level.

The Idiom, as a special language form, is greatly involved in translation, and it is very important in the language and culture of society. There are rich idioms in English and Chinese languages, and the idioms of the two languages are different. Apart from the cultural background, they are also different in the language expression of vocabulary, rhetoric devices and grammar. Because of different cultural sources, English and Chinese idioms reflect the cultural differences in the aspects of history, material life and living environment. We use translation to exchange our ideas and cultures, and through the translation, we can know more about the cultures and customs of different countries and nations. The translation of idioms has a deep influence on the level of translation and the ability of cultural communication.

\section{REFERENCES}

[1] Smith, Logn P. Words and Idioms. London: Constable and Company Ltd., 1943.

[2] F.M.Yu :A Comparative Study of English and Chinese Idioms. Shanghai : Shanghai Transportation University Press, 2001.

[3] D.A.Chen: Comparison and Translation between English and Chinese, China Translation and Publishing Corporation, 2005. (In Chinese)

[4] W.B.Chen: English Idioms and Chinese Idioms, Foreign Language Teaching and Research Press, 1992. (In Chinese)

[5] W.Z.Hu: Cross-cultural Communication and English Learning, Shanghai Translation Publishing House, 1988. (In Chinese)

[6] M c Mordie, W: English Idioms and How to Use Them, London : Oxford University Press, 1957.

[7] F.X.Wang: Culture and Language, Foreign Language Teaching and Research Press,1998. (In Chinese) 